\title{
Glasgow Blatchford Score of limited benefit for low-risk urban patients: a mixed methods study
}

\section{(ㄷ)(1) $\odot$}

Authors

David A. Leiman ${ }^{1}$, Angela M. Mills ${ }^{2}$, Frances S. Shofer ${ }^{2}$, Andrew T. Weber ${ }^{3}$, Erin R. Leiman ${ }^{4}$, Brian P. Riff ${ }^{5}$, James D. Lewis $^{6}$, Shivan J. Mehta ${ }^{6}$

Institutions

1 Division of Gastroenterology, Duke University of School of Medicine, 2301 Erwin Road, Durham, NC, USA

2 Department of Emergency Medicine, Perelman School of Medicine at the University of Pennsylvania, 3400 Spruce Street, Philadelphia, Pennsylvania, United States

3 Department of Internal Medicine, Geffen School of Medicine at the University of California at Los Angeles, 757 Westwood Plaza, Los Angeles, California, United States

4 Division of Emergency Medicine, Duke University School of Medicine, Durham, North Carolina, United States

5 Advanced Endoscopy Center, St. Jude Medical Center, Fullerton, California, United States

6 Division of Gastroenterology, Perelman School of Medicine at the University of Pennsylvania, Philadelphia, Pennsylvania, United States

submitted 23.2.2017

accepted after revision 26.6.2017

Bibliography

DOI https://doi.org/10.1055/s-0043-117880 |

Endoscopy International Open 2017; 05: E950-E958

(c) Georg Thieme Verlag KG Stuttgart · New York

ISSN 2364-3722

Corresponding author

David A. Leiman, MD, MSHP, 200 Trent Drive, Box 3913,

Durham, NC 27710

Fax: +1-919-681-8147

david.leiman@duke.edu

\section{ABSTRACT}

Background and study aims Most patients with upper gastrointestinal bleeding (UGIB) are hospitalized. Risk-stratifying UGIB with scoring tools may decrease avoidable admissions, thereby reducing the cost of care. We sought to describe how frequently low-risk UGIB patients present to urban emergency departments (ED) and the proportion who are admitted to examine how incorporating risk scores into decision support might diminish healthcare utilization in this population.

Patients and methods This is a retrospective cohort study of ED patients presenting from 2009-2013 to three urban hospitals that do not use electronic UGIB decision support. We used ED disposition diagnosis codes (ICD-9) to identify patients followed by manual chart review for verification and additional data collection. Patients with a Glasgow Blatchford Score (GBS) of 0 were classified as low risk. We also surveyed ED physicians at these hospitals to assess their beliefs about UGIB decision support.

Results Over the study period, 66 patients (13.2 per year) presented to the ED with low-risk UGIB. Of these, 10 patients $(15.2 \%)$ were admitted and none required endoscopic hemostasis. Most survey respondents $(55.6 \%, \mathrm{n}=$ 20) were aware of UGIB risk scores but a minority $(19.4 \%$, $\mathrm{n}=7$ ) used one.

Conclusions Low-risk UGIB patients infrequently present to the ED and only a minority are admitted. Despite advocacy to incorporate decision support into routine clinical care, ED physicians independently identified low risk patients. There is insufficient evidence to suggest the magnitude of this problem is large enough to warrant implementation of decision support for low risk UGIB.

\section{Introduction}

Upper gastrointestinal bleeding (UGIB) is a common clinical condition resulting in nearly 500,000 emergency department (ED) visits and almost 250,000 admissions annually in the United States [1, 2]. Prior research suggests many patients hospitalized with UGIB may be low risk for requiring an endoscopic in- tervention [3-5]. Given the challenges of appropriately triaging and managing UGIB, multiple clinical approaches have been suggested including the use of traditional endoscopy or video capsule endoscopy while patients are in the $\operatorname{ED}[6,7]$. Several clinical decision tools and risk scores also have been developed. The Glasgow Blatchford Score (GBS) specifically was created to assess patients' need for admission when presenting 
- Table 1 Glasgow Blatchford Score components.

\begin{tabular}{|c|c|}
\hline \multicolumn{2}{|l|}{ Glasgow Blatchford Score* } \\
\hline Variable & Score \\
\hline \multicolumn{2}{|l|}{ Serum BUN (mg/dL) } \\
\hline - $<18$ & 0 \\
\hline - $18-22$ & 2 \\
\hline - $23-27$ & 3 \\
\hline - $28-69$ & 4 \\
\hline . $\geq 70$ & 6 \\
\hline \multicolumn{2}{|l|}{ Hemoglobin (men, g/dL) } \\
\hline$\cdot>13$ & 0 \\
\hline - $12-13$ & 1 \\
\hline . $10-11.9$ & 3 \\
\hline.$<10$ & 6 \\
\hline \multicolumn{2}{|l|}{ Hemoglobin (women, g/dL) } \\
\hline . $>12$ & 0 \\
\hline . $10-11.9$ & 1 \\
\hline.$<10$ & 6 \\
\hline \multicolumn{2}{|l|}{ Systolic blood pressure (mmHg) } \\
\hline . >109 & 0 \\
\hline - $100-109$ & 1 \\
\hline - $90-99$ & 2 \\
\hline . $<90$ & 3 \\
\hline \multicolumn{2}{|l|}{ Others } \\
\hline - Pulse $>100$ beats per minute & 1 \\
\hline - Melena & 1 \\
\hline - Syncope & 2 \\
\hline - Liver disease & 2 \\
\hline - Heart failure & 2 \\
\hline
\end{tabular}

to the ED with complaint of UGIB and is based on routinely captured clinical data ( $\triangleright$ Table 1 ) [8].

The GBS has been validated in numerous settings and accurately identifies low risk individuals $[9,10]$. A score of zero yields a negative predictive value greater than $99 \%$ for requiring endoscopic intervention, blood transfusion, surgery or death [11]; these patients can be safely cared for without hospitalization [3]. Various professional society guidelines advocate using the GBS to identify low risk patients [2, 12-14], and an international consortium on the management of non-variceal UGIB proposed risk stratification of patients as a quality indicator [15]. However, the use of scoring tools for the triage and management of UGIB is uncommon in practice; one nation- al audit demonstrated the complete absence of its systematic use [16].

The magnitude of overuse of hospitalization for the management of patients with low risk UGIB has not been definitively characterized. As a result, it is unclear if avoidable admissions occur frequently enough to warrant implementation of decision support strategies. Some authors demonstrated that admission of patients at low risk for complications is relatively uncommon [17], while others suggest up to $16 \%$ of those admitted for UGIB may have been safely managed as outpatients [3]. Given the superior performance characteristics of the GBS in identifying low-risk patients, increased use might lead to fewer avoidable admissions.

We sought to describe the frequency at which patients present to urban EDs with low risk UGIB and the proportion of these patients who are admitted to hospital. Secondary aims included evaluating for factors associated with low-risk admissions and assessing physician awareness, perceptions of the utility, and utilization of GBS or other clinical prediction tools in the management of UGIB.

\section{Patients and methods}

\section{Study design and population}

This is a retrospective cohort study of patients presenting to the ED over a 5-year period from January 1, 2009 through December 31, 2013 at three urban hospitals with a shared electronic medical record and affiliation with an academic medical center. This time period was chosen because it occurred after both the initial description of the GBS as well as after the online publication of a prospective validation of the score [3]. All 3 hospitals have ambulatory services and deliver primary care for their local community. One hospital provides quaternary care for the region and is an emergency medicine training site with a 4-year residency program and multiple fellowship programs. The other two hospital sites serve as tertiary care referral centers.

\section{Selection of participants}

Using ICD-9 codes (Appendix 1) previously shown to reliably identify the source of UGIB in patients admitted to the hospital, we screened patients for inclusion from the health system's database [18]. Patients were eligible for inclusion if one or more of their first 4 ED final diagnoses was compatible with UGIB. Patients were excluded if they were under age 18, did not report symptoms of upper Gl bleeding (melena, hematemesis or coffee ground emesis), or had a GBS of 1 or more. In order to meet criteria for final analysis, patients were required to have a documented history of UGIB in the electronic medical record (EMR) such as hematemesis or coffee ground emesis.

Initial review excluded those with vital sign or laboratory abnormalities that would preclude them from being low risk using a standardized abstraction form. Among the remaining eligible records, clinical data were manually abstracted using a standardized form and defined variables from the EMR and in accordance with methodologic guidelines for chart review [19]. Abstractors were trained and performance was monitored. All 
three hospitals use a proprietary EMR to document ED care and this was reviewed in full. No relevant data were missing. If physical exam or important history elements were not specifically documented as being present in the medical chart, for example melena or syncope, these were considered absent or not to have occurred. Ambiguous elements were resolved through consensus by abstractors. Inpatient medical record review was also performed for all patients admitted to the hospital.

A second component of our study included conducting a survey of attending ED physicians $(n=72)$ at the three hospitals to assess perceived usefulness and self-reported utilization of scoring tools for the management of UGIB. Admission decisions in the health system are made by attending level ED providers. All attending physicians currently working at the three hospitals were eligible unless they participated in the design of this research. Physicians were contacted via email to participate (SurveyMonkey.com, Palo Alto, CA) in a 6-question survey (Appendix 2). Non-responders were emailed up to two additional times to solicit responses.

\section{Methods and measurements}

We collected standard demographic information (age, sex and race), components of the Charlson Comorbidity Index (CCI), outpatient medications as documented in the ED EMR (proton pump inhibitors [PPI], histamine-2-receptor antagonists [H2RA], selective serotonin reuptake inhibitors [SSRI], glucocorticoids, aspirin, non-steroidal anti-inflammatory drugs [NSAID], warfarin, antiplatelet agents, low molecular weight heparin, direct thrombin inhibitors and factor Xa inhibitors), use of alcohol within 24 hours of presentation, insurance status, time and day of presentation, ED disposition, hospital location and if a resident or physician extender was involved in care. The computed Charlson Comorbidity Index score represents a modification of the original scale because we did not assign points for history of peptic ulcer disease given that was a factor being investigated as part of the presentation [20].

Resource utilization estimates for patients who were not discharged from the ED included disposition level of care, use of intravenous PPI, gastroenterology consultation, endoscopy, use of procedural sedation, endoscopic findings and hospital length of stay (LOS).

\section{Outcomes}

The primary outcome in our study was ED disposition. Patients were categorized as discharged, leaving against medical advice, hospital admission or placement in observation. Secondary outcomes included healthcare utilization estimates for patients not discharged from the ED, factors associated with avoidable admissions and endoscopic findings in low risk patients who underwent upper endoscopy.

For the survey component, perceived usefulness was rated on a 6-point Likert-like scale.

\section{Analysis}

Descriptive statistics were calculated for all variables, including mean and standard deviation for continuous variables and frequencies for categorical values. Differences between patients

2,183 patients identified by electronic screening of ICD-9 code

34 patients excluded (age $<18$ years)

2,149 patients screened

$1,463$ patients excluded ( $G B S \geq 1)$

$\vee$

686 patients with $\mathrm{GBS}=0$

620 patients excluded (no UGIB complaint)

66 patients analyzed

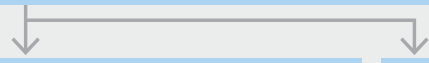

10 patients admitted or observation

56 patients discharged

- Fig. 1 Study recruitment flowchart. Patients were screened based on emergency department disposition diagnosis codes (ICD-9) and verified by manual review of the electronic medical record.

who were and were not discharged from the ED were assessed using a $\mathrm{X}^{2}$ test for categorical variables and a $t$-test or MannWhitney $U$-test for continuous variables. The Kruskal-Wallis test was used to examine the association between providers' perceived utility of UGIB scoring tools and other factors such as years in practice, practice location and others.

All statistical analyses were performed with STATA software (version 13.0; StataCorp, College Station, TX). P values less than 0.05 were considered significant. All components of this study were approved by the institutional review board at the University of Pennsylvania.

\section{Results}

\section{Characteristics of study subjects}

There were 2,183 patients identified by ICD-9 disposition diagnoses from the ED over the study period. Among these, 2,117 were excluded because they were not low risk based on a GBS $\geq 1$, did not have documented evidence of UGIB or their age was $<18$ ( $\triangleright$ Fig. 1 ). The remaining 66 patients were ultimately included in the final analysis ( $\triangleright$ Table 2 ). The included patient population was young with a mean age of $34.5 \pm 12.8$ years. Nearly all $(95.5 \%, n=63)$ had a computed Charlson Comorbidity Index of 0 and were infrequently $(15.2 \%, n=10)$ prescribed medications that have been shown to be associated with UGIB such as aspirin or NSAIDs.

\section{Admitted patients}

Ten (15.2\%) of the 66 patients with GBS $=0$ were admitted or placed in observation. The mean age of these patients was similar to those patients who were discharged (39.9 vs. 33.5 years; 
- Table2 Baseline patient characteristics.

\begin{tabular}{|c|c|c|c|c|c|}
\hline & \multicolumn{2}{|c|}{ Admits/Observations $(n=10)$} & \multicolumn{2}{|c|}{ Discharges $(n=56)$} & \multirow[t]{2}{*}{$P$ value } \\
\hline & $\mathbf{n}$ & $(\%)$ & $\mathbf{n}$ & (\%) & \\
\hline Age (mean $\pm S D)$ & \multicolumn{2}{|c|}{$39.9 \pm 14.8$} & \multicolumn{2}{|c|}{$33.5 \pm 12.3$} & 0.15 \\
\hline Female & 6 & $(60.0)$ & 25 & $(44.6)$ & 0.50 \\
\hline Black & 7 & $(70.0)$ & 39 & $(69.6)$ & 0.65 \\
\hline Uninsured & 2 & $(20.0)$ & 19 & (33.9) & 0.38 \\
\hline Attending only & 6 & $(60.0)$ & 19 & $(33.9)$ & 0.12 \\
\hline Hospital & & & & & 0.70 \\
\hline - Quaternary care center & 3 & $(30.0)$ & 24 & $(42.9)$ & \\
\hline - Tertiary care center 1 & 4 & $(40.0)$ & 16 & $(28.6)$ & \\
\hline - Tertiary care center 2 & 3 & $(30.0)$ & 16 & $(28.6)$ & \\
\hline \multicolumn{6}{|l|}{ Time Seen } \\
\hline - Night & 10 & $(100.0)$ & 35 & $(62.5)$ & 0.02 \\
\hline - Weekend & 4 & $(40.0)$ & 17 & $(30.4)$ & 0.55 \\
\hline $\mathrm{CCl} \geq 2$ & 0 & $(0.0)$ & 53 & $(94.6)$ & 0.45 \\
\hline \multicolumn{6}{|l|}{ Medications } \\
\hline - Proton pump inhibitor & 2 & $(20.0)$ & 10 & (17.9) & 0.87 \\
\hline - Aspirin & 0 & $(0.0)$ & 3 & (5.4) & 0.45 \\
\hline
\end{tabular}

$P=0.15)$. Presentation at night was statistically significantly associated with admission with $100 \%(n=10)$ of patients admitted between the hours of 5pm and 7am, while only 24 (42.9\%) discharges occurred during this timeframe $(P=0.02)$. Sixty percent of admissions were female compared to $44.6 \%$ of discharges but this was not statistically significant. There were a similar proportion of black patients in each group. Neither insurance status, resident or physician extender involvement, hospital location, presentation over the weekend, baseline use of PPI nor aspirin were associated with being admitted.

\section{Resource utilization}

Of the 10 patients who were not discharged home from the ED, 2 were placed on observation and 8 admitted to a standard care bed; no patients were triaged to intensive care. The median LOS among these patients was 1.5 days. There was no significant difference in LOS between those placed in observation and those admitted to a standard care bed or among those who received a gastroenterology consult. Two admitted patients received intravenous PPI. All patients were eventually discharged to home.

A total of 6 patients $(9.1 \%$ overall, $60 \%$ of non-discharged patients) underwent endoscopy, including 1 in a patient who was placed on observation and 5 who were admitted. There was no difference in median hospital LOS among these two groups $(P=0.64)$. Every patient who had a gastroenterology consult ultimately underwent endoscopy $(P=0.002)$.
No patients undergoing endoscopy required an endoscopic intervention for hemostasis, received any red blood cell transfusions, had surgery or died. The majority of endoscopic findings were gastritis and esophagitis ( $>$ Table 3 ). All endoscopy was performed in an endoscopy suite during daytime hours with propofol sedation.

\section{Survey results}

There were 72 eligible attendings of whom 36 (50\%) completed the survey. The majority of responders were male $(n=22$, $61.1 \%)$ and located at the quaternary care site $(n=24,66.7 \%)$. Most respondents indicated awareness of risk scores for UGIB $(n=20,55.6 \%)$ but only a minority $(n=7,19.4 \%)$ reported using a risk score within that same time period. In contrast, nearly all respondents $(n=33,94.1 \%)$ reported using a risk tool for the management of conditions other than UGIB. There was no difference in mean number of years of independent practice $(P=0.64)$ or self-recalled number of patients with UGIB treated within the preceding 6 months $(P=0.82)$ between those who were aware of scoring tools to manage UGIB and those who were not ( $\triangleright$ Table 4$)$.

Overall, most $(n=21,62 \%)$ respondents perceived risk scores for UGIB to be at least slightly useful in the management of patients with UGIB in the ED ( $>$ Fig. 2). There was no significant association seen between sex, practice location, years of independent practice, recalled number of patients with UGIB, awareness of UGIB scoring tools, use of UGIB or non-UGIB scor- 
- Table 3 Endoscopic findings.

\begin{tabular}{|l|l|l|l|}
\hline \multirow{2}{*}{ Patient } & Endoscopic findings & Small bowel \\
\hline 1 & Esophagus & Stomach & Normal \\
\hline 2 & Hiatal hernia with ring & Small antral erosion & Normal \\
\hline 3 & Esophagitis & $\begin{array}{l}\text { 1. Antral and fundic erythema } \\
\text { 2. Multiple gastric body and fundic erosions } \\
\text { 3. Few small<4 mm clean based fundic ulcers }\end{array}$ & Normal \\
\hline 4 & $\begin{array}{l}\text { 1. Gastric bypass anatomy } \\
\text { 2. Ulceration at anastomosis }\end{array}$ & Normal \\
\hline 5 & $\begin{array}{l}\text { 1. Gastritis in body } \\
\text { 2. Fundic erosion }\end{array}$ & Superficial duodenal bulb ulcers \\
\hline 6 & Normal & $\begin{array}{l}\text { 1. Ulceration in antrum } \\
2 . \text { Small body and antral erosions }\end{array}$ & Normal \\
\hline
\end{tabular}

- Table 4 Survey results by question.

\begin{tabular}{|c|c|c|c|}
\hline \multirow[t]{2}{*}{ Question ( $n=$ total number of responses) } & \multicolumn{2}{|c|}{$\begin{array}{l}\text { Are you aware of any decision support } \\
\text { tools or risk scores to help in the manage- } \\
\text { ment of patient with UGIB in the ED? }\end{array}$} & \multirow[t]{2}{*}{$P$ value } \\
\hline & Yes $(n=20)$ & No $(n=16)$ & \\
\hline $\begin{array}{l}\text { How long have you been in practice as an attending (years since finishing residency)? } \\
(n=36)\end{array}$ & 13.3 years & 11.8 years & 0.64 \\
\hline $\begin{array}{l}\text { How many patients with UGIB do you think you have managed in the last six months? } \\
(\mathrm{n}=36)\end{array}$ & 7.8 patients & 8.3 patients & 0.82 \\
\hline $\begin{array}{l}\text { Have you used any decision support tools or risk scores to help in the management of } \\
\text { patients with UGIB in the last six months? }(n=18)\end{array}$ & Yes: $38.9 \%(n=7)$ & $\mathrm{N} / \mathrm{A}$ & $\mathrm{N} / \mathrm{A}$ \\
\hline $\begin{array}{l}\text { On a scale of } 1-6 \text { (with } 1 \text { being not useful and } 6 \text { being extremely useful), how useful } \\
\text { do you think decision support tools or risk scores are for the management of UGIB in } \\
\text { the emergency department? }(n=34)\end{array}$ & $\begin{array}{l}4^{*} \\
(n=18)\end{array}$ & $\begin{array}{l}4^{*} \\
(n=16)\end{array}$ & 0.88 \\
\hline $\begin{array}{l}\text { Do you use decision support tools or risk scores in the management of conditions } \\
\text { other than UGIB? }(n=33)\end{array}$ & Yes: $88.9 \%(n=16)$ & Yes: $100 \%(n=16)$ & 0.49 \\
\hline
\end{tabular}

ing tools and perceived usefulness. When respondents who were aware of tools for the management of UGIB were asked to identify which tool they were familiar with, a majority $(n=$ $11,65 \%)$ named the GBS.

\section{Discussion}

This study provides an examination of healthcare utilization among patients presenting to the ED with UGIB who are low risk by GBS criteria. We found that only a small minority of patients presenting to the ED with complaints of UGIB had a GBS of 0 and few of them were admitted. Only night-time presentation was associated with low-risk admission. Despite ED physicians' awareness of - and belief that there might be value in applying - risk tools for managing UGIB, they report rarely using them. Although some have argued for the broader use of decision support like the GBS in managing patients with UGIB [21], implementing its use in the ED to specifically identify the few low-risk patients who present does not seem to justify the costs and organizational effort required, especially with many competing needs to improve value of care.

GBS is consistently superior to other tools in identifying lowrisk patients [4, 5, 22]. It also has been shown that higher GBS at admission is associated with recurrent bleeding after discharge [23]. Our study aimed to evaluate only patients with the lowest risk of needing endoscopic intervention $(G B S=0)$ who should be safely discharged from the ED. Although it is readily available and easy to calculate, patients with low risk UGIB are still admitted. No systematic investigation of why such patients are admitted has been undertaken. Several previous studies 


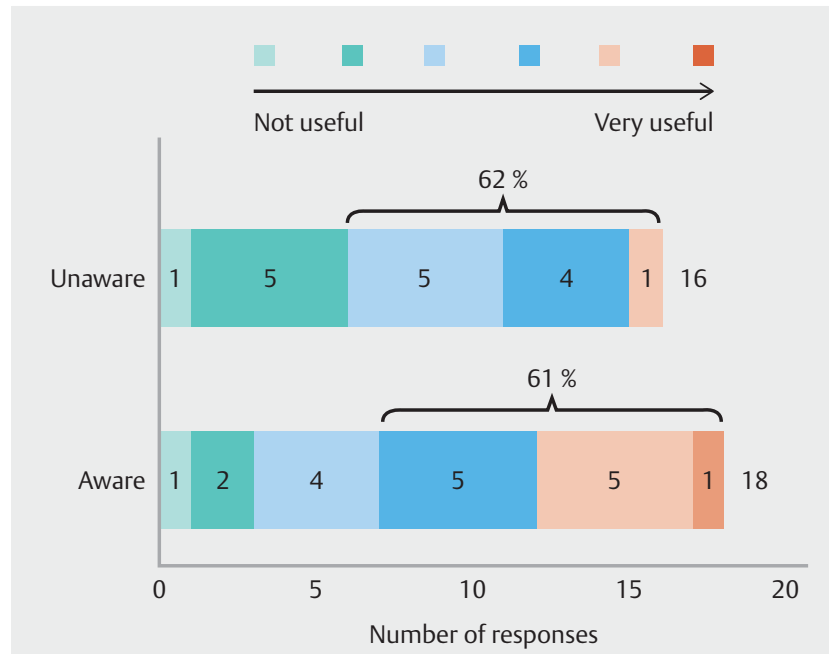

- Fig. 2 Thirty-four physicians rated their perceived utility for risk scores in the management of upper gastrointestinal bleeding. The median response level was 4 in both groups of physicians suggesting a perceived usefulness for such decision support.

have examined barriers to, and attempted to improve, adherence to practice guidelines recommending the use of risk scores for UGIB $[24,25]$. These studies were focused at practitioner rather than patient level factors. We attempted to identify such patient factors and among our cohort found only presentation at night to be significantly associated with admission. This is consistent with real-life and one likely explanation is related to patient volume. Some have shown ED censuses are highest on off hours, which is also a time when there is an increased risk of leaving without being seen [26, 27]. It is plausible that to make up for the increased patient burden during night-time hours and to avoid patients leaving without being seen, even those with low-risk features may be admitted to help with throughput. Non-business hours are also times when outpatient clinics are rarely open, so it may be that patients presenting at night are suspected of being most vulnerable and to ensure follow-up, would be more likely to be admitted $[28,29]$.

Even with the night-time admissions, ED physicians in our study made appropriate triage decisions on patients with lowrisk UGIB most of the time without relying on GBS or other decision support tools. Both rates of admission (15.2\%) and selfreported use of decision support (19.4\%) were low. This suggests that most providers are accurately risk-stratifying patients intuitively. Although not restricted to low-risk patients, a previous analysis found ED physician accuracy in predicting need for endoscopic intervention was superior based on clinical decision-making alone compared to using the GBS [30]. In our study, endoscopic hemostasis was not required in any case but importantly, none of the patients were admitted to an intensive care unit. This fact may indicate ED physician suspicion for the need for hemostasis was not high.

Previous studies, including many from Europe, have demonstrated that a high percentage of low-risk UGIB patients are ad- mitted $[3,10,31]$. A more recent analysis from New Zealand found only $1 \%$ of patients presenting to the ED with complaints of UGIB had a GBS of 0 [32]; an earlier study in the United States found only $4 \%$ of patients admitted for management of UGIB were low risk by GBS criteria [17]. In our study, few patients with low-risk UGIB presented to the ED, were admitted and ultimately underwent endoscopic evaluation. While theoretically avoidable, the magnitude of patients who were admitted is underwhelming. In contrast, it is possible that some higher-risk patients with $G B S \geq 1$ were discharged, although the primary aim of this study was to evaluate avoidable admissions rather than focus on potentially inappropriate discharges. Although the latter was not evaluated as part of this study, it may represent an area for future study. Also, the optimal number of admissions may not be zero especially if there are other compelling reasons to admit patients.

This is a retrospective analysis performed across 3 EDs in a single urban health system, which could affect the profile of patients in our study. During this period, there were nearly 700,000 ED patient visits across the 3 hospital sites, therefore, less than $0.1 \%$ of patients presented with low-risk UGIB and even fewer were admitted. Given the breadth of patients seen at the included hospitals, the population should be representative of patients presenting with UGIB to urban EDs. To maximize our capture of patients presenting with low-risk UGIB, we screened for inclusion using ED discharge coding. The ICD-9 codes used have previously been shown to accurately identify inpatients with UGIB. As with prior studies, we included subcodes compatible with UGIB but that did not specifically mention hemorrhage [33]. Further, we did not restrict our codes to the primary diagnosis, recognizing that the ICD-9 is assigned by a third-party coder and may not always reflect the principal clinical impression of the clinician. Although a potential limitation, no documentation describing the presence of melena was considered a surrogate for its absence in our study. Because melena would justify stratifying patients as non-low risk, in order not to inadvertently affect the specificity of patients in our cohort we only assigned GBS points when definitively documented in the medical record. Notably, we found large discordance between patients with true complaints of UGIB $(n=66)$ based on manual chart review and those classified by ICD-9 code $(n=682)$, suggesting that future studies focusing on UGIB in the ED may need to rely on manual record review or other novel search criteria to accurately identify cases.

To reduce the overall number of avoidable admissions for UGIB, some have suggested increasing use of GBS in practice. $A$ recent national survey of American physicians revealed knowledge and application of scoring tools is low and advocated developing systems to increase their use [21]. In our survey, respondents' awareness of GBS was high but their use of it remained infrequent, although we acknowledge this may be influenced by the fact that our response rate was $50 \%$ and that the majority of responders were from the quaternary care center. Despite this, few patients with a GBS of 0 were admitted. It is possible that physicians staffing these EDs have different knowledge or beliefs about decision tools than physicians from other practice settings. Even still, it is hard to justify the expen- 
diture and potential decision-support fatigue to create such systems given the small number of low-risk UGIB patients who are seen in general and even smaller number who are admitted [34]. While there may always be room for improvement, we observed a rate of only 2 avoidable admissions per year, making it difficult to suggest that more uniform application of GBS would substantially change healthcare expenditures. A more useful approach may be to raise the threshold for low-risk gastrointestinal bleeding, which has been previously studied and advocated for in more recent European guidelines [12, 32, 35-37]. Although we would likely have found more low-risk patients at a higher threshold of a GBS of 1, it would come at the cost of reducing the near perfect discrimination of a GBS of 0 for needing interventions.

\section{Conclusion}

In summary, this represents a comprehensive attempt to describe the frequency of ED presentation, healthcare utilization and factors associated with avoidable admissions in patients with lowest-risk UGIB defined by GBS. Although avoidable admissions occur, the overall magnitude of this problem is small. Using GBS to identify high-risk patients in need of inpatient evaluation is desirable. However, contrary to existing guidelines, the routine use of GBS - or even the creation of new decision support tools - for discriminating low-risk UGIB patients in the current ED setting would provide a limited economic benefit. Given that avoidable admissions are not a major driver of the cost of care for UGIB, future efforts at reducing healthcare costs should focus on increasing efficiency of the care delivered in hospital and expanding the pool of patients that can be defined as "low risk" and suitable for outpatient management.

\section{Acknowledgements}

This work was supported by the NIH/NIDDK T32 DK007740 (DAL) and K24-DK078228 (JDL).

Competing interests

None

\section{References}

[1] Myer PA, Mannalithara A, Singh G et al. Clinical and economic burden of emergency department visits due to gastrointestinal diseases in the United States. Am J Gastroenterol 2013; 108: 1496-1507

[2] Hwang JH, Fisher DA, Ben-Menachem T et al. The role of endoscopy in the management of acute non-variceal upper Gl bleeding. Gastrointest Endosc 2012; 75: $1132-1138$

[3] Stanley AJ, Ashley D, Dalton HR et al. Outpatient management of patients with low-risk upper-gastrointestinal haemorrhage: multicentre validation and prospective evaluation. Lancet 2009; 373: 42-47

[4] Laursen SB, Hansen JM, Schaffalitzky de Muckadell OB. The Glasgow Blatchford Score is the most accurate assessment of patients with upper gastrointestinal hemorrhage. Clin Gastroenterol Hepatol 2012; 10: $1130-1135$ e 1

[5] Chen IC, Hung MS, Chiu TF et al. Risk scoring systems to predict need for clinical intervention for patients with nonvariceal upper gastrointestinal tract bleeding. Am J Emerg Med 2007; 25: 774 - 779

[6] Meltzer AC, Ali MA, Kresiberg RB et al. Video capsule endoscopy in the emergency department: a prospective study of acute upper gastrointestinal hemorrhage. Ann Emerg Med 2013; 61: 438 - 443 e1

[7] Lee JG, Turnipseed S, Romano PS et al. Endoscopy-based triage significantly reduces hospitalization rates and costs of treating upper GI bleeding: a randomized controlled trial. Gastrointest Endosc 1999; 50: $755-761$

[8] Blatchford O, Murray WR, Blatchford M. A risk score to predict need for treatment for uppergastrointestinal haemorrhage. The Lancet 2000; 356: $1318-1321$

[9] Stanley A]. Update on risk scoring systems for patients with upper gastrointestinal haemorrhage. World J Gastroenterol 2012; 18: $2739-2744$

[10] Stephens JR, Hare NC, Warshow U et al. Management of minor upper gastrointestinal haemorrhage in the community using the Glasgow Blatchford Score. Eur J Gastroenterol Hepatol 2009; 21: 1340 - 1346

[11] Pang SH, Ching JY, Lau JY et al. Comparing the Blatchford and preendoscopic Rockall score in predicting the need for endoscopic therapy in patients with upper $\mathrm{Gl}$ hemorrhage. Gastrointest Endosc 2010; 71: $1134-1140$

[12] Gralnek IM, Dumonceau JM, Kuipers EJ et al. Diagnosis and management of nonvariceal upper gastrointestinal hemorrhage: European Society of Gastrointestinal Endoscopy (ESGE) Guideline. Endoscopy 2015; 47: a $1-46$

[13] Barkun ABM, Kuipers E, Sung J et al. International Consensus Recommendations on the Management of Patients With Nonvariceal Upper Gastrointestinal Bleeding. Ann Intern Med 2010; 152: 101 - 113

[14] Greenspoon J, Barkun A, Bardou M et al. Management of patients with nonvariceal upper gastrointestinal bleeding. Clin Gastroenterol Hepatol 2012; 10: $234-239$

[15] Kanwal F, Barkun A, Gralnek IM et al. Measuring quality of care in patients with nonvariceal upper gastrointestinal hemorrhage: development of an explicit quality indicator set. Am J Gastroenterol 2010; 105: $1710-1718$

[16] Bensoussan K, Fallone C, Barkun A et al. A sampling of Canadian practice in managing nonvariceal upper gastrointestinal bleeding before recent guideline publication: Is there room for improvement? Can J Gastroenterol Hepatol 2005; 19: 487-495

[17] Piccoli L, Gilrain K, Holliday N et al. 411 Is the Glasgow-Blatchford Bleeding Score Useful in Reducing Upper Gastrointestinal Bleed Admissions in a United States Hospital? Ann Emerg Med 2011; 58: S317-S8

[18] Cooper GS, Chak A, LLoyd LE et al. The accuracy of diagnosis and procedural codes for patients with upper $\mathrm{Gl}$ hemorrahge. Gastrointest Endosc 2000; 51: $423-426$

[19] Kaji AH, Schriger D, Green S. Looking through the retrospectoscope: reducing bias in emergency medicine chart review studies. Ann Emerg Med 2014; 64: 292 - 298

[20] Charlson M, Pompei P, Ales K et al. A new method of classifying prognostic comorbidity in longitudinal studies: development and validation. J Chronic Dis 1987; 40: 373-383

[21] Liang PS, Saltzman JR. A national survey on the initial management of upper gastrointestinal bleeding. J Clin Gastroenterol 2014; 48: e9398

[22] Wang $\mathrm{CH}$, Chen YW, Young YR et al. A prospective comparison of 3 scoring systems in upper gastrointestinal bleeding. Am J Emerg Med 2013; 31: $775-778$ 
[23] Sengupta N, Tapper EB, Patwardhan VR et al. High Glasgow Blatchford Score at admission is associated with recurrent bleeding after discharge for patients hospitalized with upper gastrointestinal bleeding. Endoscopy 2016; 48: 9-15

[24] Barkun AN, Hawes IA, Armstrong D et al. M1079 Improving Adherence to Guidelines When Managing Non-Variceal Upper Gastrointestinal Bleeding: A National Cluster Randomized Trial of a Multifaceted Strategy. Gastroenterology 2010; 138: S-327

[25] Hayes SM, Murray S, Dupuis M et al. Barriers to the implementation of practice guidelines in managing patients with nonvariceal upper gastrointestinal bleeding: A qualitative approach. Can J Gastroenterol 2010; 24: $289-296$

[26] Pitts SR, Niska RW, Xu J et al. National Hospital Ambulatory Medical Care Survey: 2006 Emergency Department Summary. Natl Health Stat Repor 2008; 6: 1 - 38

[27] Mohsin M, Forero R, leraci S et al. A population follow-up study of patients who left an emergency department without being seen by a medical officer. Emerg Med J 2007; 24: 175-179

[28] Grumbach K, Keane D, Bindman A. Primary care and public emergency department overcrowding. Am J Public Health 1993; 83: $372-378$

[29] Magid DJ, Wang Y, Herrin J et al. Relationship between time of day, day of week, timeliness of reperfusion, and in-hospital mortality for patients with acute st-segment elevation myocardial infarction. JAMA 2005; 294: $803-812$

[30] Farooq FT, Lee MH, Das A et al. Clinical triage decision vs risk scores in predicting the need for endotherapy in upper gastrointestinal bleeding. Am J Emerg Med 2012; 30: 129-134
[31] Schiefer M, Aquarius M, Leffers P et al. Predictive validity of the Glasgow Blatchford Bleeding Score in an unselected emergency department population in continental Europe. Eur J Gastroenterol Hepatol 2012; 24: $382-387$

[32] Laursen SB, Dalton HR, Murray IA et al. Performance of new thresholds of the Glasgow Blatchford Score in managing patients with upper gastrointestinal bleeding. Clin Gastroenterol Hepatol 2015; 13: 115 121 e 2

[33] Dulai GS, Gralnek IM, Oei TT et al. Utilization of health care resources for low-risk patients with acute, nonvariceal upper GI hemorrhage: an historical cohort study. Gastrointest Endosc 2002; 55: 321 - 327

[34] Horsky J, Schiff GD, Johnston D et al. Interface design principles for usable decision support: a targeted review of best practices for clinical prescribing interventions. J Biomed Inform 2012; 45: $1202-1216$

[35] June IL, Gordon A, Farrugia D et al. Safe discharge of patients with low-risk upper gastrointestinal bleeding (UGIB): can the use of Glasgow-Blatchford Bleeding Score be extended? Acute Med 2011; 10: $176-181$

[36] Stanley AJ, Laine L, Dalton HR et al. Comaprison of scoring systems for patients presenting with upper gastrointestinal bleeding: international multicentre prospective study. BMJ 2017; 356: i6432

[37] Mustafa Z, Cameron A, Clark AE et al. Outpatient management of low-risk patients with upper gastrointestinal bleeding: can we safely extend the Glasgow Blatchford Score in clinical practice? Eur J Gastroenterol Hepatol 2015; 27: 512 - 515 


\section{Appendix 1}

\section{List of ICD-9 codes utilized}

$530.10,530.11$ (esophagitis with or without mention of hemorrhage)

530.70 (Mallory-Weiss syndrome)

530.82 (esophageal hemorrhage)

531.xx (gastric ulcer)

532.xx (duodenal ulcer)

533.xx (peptic ulcer)

534.xx (gastrojejunal ulcer)

535.xx (gastritis or duodenitis)

537.83 (angiomata of stomach or duodenum)

578.00 (hematemesis)

578.10 (melena)

578.90 (hemorrhage of the GI tract, unspecified)
Appendix 2

Physician survey querying usage patterns

of and attitudes toward decision support

in upper gastrointestinal bleeding

\section{Background}

1. How long have you been in practice as an attending (years since finishing residency)?

2. How many patients with upper gastrointestinal bleeding (UGIB) do you think you have managed in the last six (6) months?

\section{Decision Support}

3a. Are you aware of any decision support tools or risk scores to help in the management of patients with UGIB in the emergency department? (yes/no)

3b. Which decision support tools are you aware of?

4a. Have you used any decision support tools or risk scores to help in the management of patients with UGIB in the last six (6) months? (yes/no)

4b. Which decision support tools or risk scores have you used? 5. On a scale of $1-6$ (with 1 being not useful and 6 being extremely useful), how useful do you think decision support tools or risk scores are for the management of UGIB in the emergency department? (yes/no)

6 a. Do you use decision support tools or risk scores in the management of conditions other than UGIB? (yes/no)

$6 \mathrm{~b}$. Which decision support tools or risk scores do you use? 